\title{
MEASUREMENT ERROR WITH DIFFERENT COMPUTER VISION TECHNIQUES
}

\author{
O. Icasio-Hernández ${ }^{\mathrm{a}, \mathrm{b}} *$ Y. I. Curiel-Razo ${ }^{\mathrm{b}}$, C. C. Almaraz-Cabral ${ }^{\mathrm{b}}$, S. R. Rojas-Ramirez ${ }^{\mathrm{b}}$, J.J. González-Barbosa ${ }^{\mathrm{b}}$ \\ ${ }^{a}$ Centro Nacional de Metrología, km. 4.5 carretera a los Cués, el Marqués, Qro., México. - oicasio@cenam.mx \\ ${ }^{\mathrm{b}}$ Instituto Politécnico Nacional (CICATA), Cerro Blanco No. 141, Colinas del Cimatario Qro., México. - \\ (yajaira.curielrazo, cesarcruzalmaraz,gonzbarji)@gmail.com, sergio.rojas@unaq.edu.mx.
}

KEY WORDS: Estimation error, Image reconstruction, Measurement techniques, Measurement uncertainty

\begin{abstract}
:
The goal of this work is to offer a comparative of measurement error for different computer vision techniques for 3D reconstruction and allow a metrological discrimination based on our evaluation results. The present work implements four 3D reconstruction techniques: passive stereoscopy, active stereoscopy, shape from contour and fringe profilometry to find the measurement error and its uncertainty using different gauges. We measured several dimensional and geometric known standards. We compared the results for the techniques, average errors, standard deviations, and uncertainties obtaining a guide to identify the tolerances that each technique can achieve and choose the best.
\end{abstract}

\section{INTRODUCTION}

Several imaging techniques for accurate 3D reconstruction and their quality is improving rapidly. Some authors like (Hocken, 2011), (Maier-Hein, 2013), (Rashmita, 2013) and (Ng, 2011) believe that an evaluation is necessary for these techniques, so they assess the process algorithms to determine the quality of the measurements.

The purpose of this work is to assess the measurement error of passive stereoscopy, active stereoscopy, shape from contour and fringe profilometry using different known dimensional gauges. The techniques considered apply algorithms to fit a set of points from a gauge image to a particular geometry. The gauge measurement error define the quality of the technique. Authors as (Rashmita, 2013), (Ng, 2011) and (Salvi, 2007) suggest that industry can use these techniques for quality control if the measurement error and its uncertainty is under the verified tolerances.

The gauge measurement error is defined as the measure calculated with the technique minus the known value of the gauge. The nominal used lengths were $3 \mathrm{~mm}, 50 \mathrm{~mm}$ and $100 \mathrm{~mm}$ block gauges, a $10 \mathrm{~mm}$ metallic pin diameter, and a height of 15.081 $m m$ of fine-grained 3D printed piece of gypsum, see Figure 1.

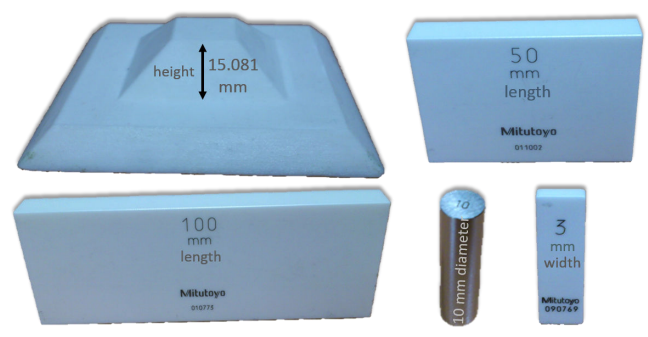

Figure 1. Gauges used to measure the error of different optical measurement techniques.

The closest work to our own investigations has been proposed by (Ramos,2011), who instead of implemented techniques used different instruments with digitization techniques incorporated into

\footnotetext{
${ }^{*}$ Corresponding author
}

its measurement software and similar to our work he used different reference gauges. Most of the reported vision techniques in (Hocken, 2011), (Maier-Hein, 2013), (Rashmita, 2013), (Ng, 2011) and (Salvi, 2007) offer measurement errors using objects with no traceability. Our work report measurement errors using gauges with traceability to national standards.

This paper is organized as follows. Section 2 deals with the uncertainty applied to the results. In section 3 , we implemented the $3 \mathrm{D}$ reconstruction techniques including references, calibration process, the particular experimental setup and the results. The comparative results between techniques are given in section 4 and section 5 deals with the conclusions and discussion about results.

\section{UNCERTAINTY}

\subsection{Uncertainty applied on results}

In accordance with (ISO 14253-1, 1998) every measurement process must take into account the accuracy (ISO 14253-1, 1198) represented by a resultant error and its corresponding uncertainty. We based the uncertainty calculation on the guide to the expression of uncertainty in measurement (GUM, 2010) and (ISO 15530-3, 2011) Coordinate Measuring Machines (CMM): Technique for determining the uncertainty of measurement Part 3: Use of calibrated work pieces or measurement standards (ISO 15530-10, 2011). The expanded uncertainty expression $U$ for our error measurand is:

$$
U=k \cdot u_{c}(y)=k \cdot \sqrt{u_{c a l}^{2}+u_{p}^{2}+u_{b}^{2}+u_{w}^{2}}
$$

where $k$ is the coverage factor with a conventional value of two to achieve a probability of $94.5 \%$ of confidence in the calculated uncertainty. $u_{c}(y)$ is the combined uncertainty of each contributor $y . u_{c a l}$ is the standard calibration uncertainty reported in the certificate of calibration of the gauge reference used. $u_{p}$ is the standard uncertainty associated with the measurement process, it includes sources of uncertainty as repeatability, the measurement environment, subjections, measurement strategy and procedure, 
speed of measurement, and geometry of the measuring system. $u_{b}$ is the standard uncertainty associated with the systematic error $b$ of the measurement process evaluated using the calibrated work piece, in our case the systematic error is the measurand so $u_{b}$ will not be considered. $u_{w}$, is the standard uncertainty of the material and the variations of manufacture, it includes sources of uncertainty as variations in thermal expansion, errors of shape, roughness, elasticity, and plasticity.

Due to the error ranges of the implemented techniques and the quality of the gauges $u_{c a l}, u_{b}$ and $u_{w}$ are considered zero so Equation 1 is simplified as follows:

$$
U=k \cdot \sqrt{u_{p}^{2}}
$$

From Equation 2, the uncertainty $u_{p}$ is Equation3:

$$
u_{p}=\frac{\sigma_{x}}{\sqrt{n}}
$$

where $\sigma_{x}$ the standard deviation of the measurements defined in Equation 4 , and $n$ is the number of measurements.

$$
\sigma_{x}=\sqrt{\frac{\sum_{j=1}^{n}\left(q_{j}-\bar{q}\right)^{2}}{n-1}} ;
$$

where $n$ is the number of measurements, $q_{j}$ is the $j$ measurement and $\bar{q}$ is the mean of the measurements.

All the techniques were implemented in a laboratory with a temperature of $20^{\circ} \pm 3^{\circ} \mathrm{C}$, with artificial illumination and the absence of direct sunlight. The number of measurements made with each technique is at least $n=3$ to calculate uncertainty. The uncertainties of calibration certificate for the gauges used are in the range of $\pm 12 \mathrm{~nm}$ to $\pm 250 \mathrm{~nm}$ which, as mention before, were consider as approximately zero for the case of the implemented techniques.

\section{3D RECONSTRUCTION TECHNIQUES}

\subsection{Passive Stereoscopy Technique (PST)}

According with (Szeliski, 2010), in computer vision, the topic of Passive Stereoscopy Technique (PST), specially stereo matching, has been one of the most widely studied and fundamental problems, and continues to be one of the most active research areas. While photogrammetric matching concentrated mainly on aerial imagery, computer vision applications include modeling the human visual system, robotic navigation and manipulation, as well as view interpolation and image-based rendering, 3D model building, and mixing live action with computer-generated imagery.

3.1.1 Calibration method and model for PST To calibrate the cameras we considered the model proposed by (Hall, 1982) and (Tsai, 1987) both uses the pinhole camera model. This model is extended with some correction parameters, such as radial lens distortion and tangential lens distortions that causes radial and tangential displacement in the image plane.

Camera calibration means the process of determining the internal camera geometric and optical characteristics (intrinsic parameters) and/or the 3D position and orientation of the camera frame relative to a certain world coordinate system (extrinsic parameters). The purpose of the calibration is to set a relationship between 3D points from the world and 2D points from the image, seen by the computer (Tsai, 1987), (Heikkila, 1997), and (Vilaca, 2010).

We performed cameras calibration for PST with the toolbox from (Bouguet, 2013) that uses a checkerboard calibration pattern. Table 1 shows the results of the intrinsic parameters for each camera and its uncertainties.

Table 1. Camera calibration results for Passive and Active Stereoscopy Techniques.

\begin{tabular}{ccccc}
\hline Value & Left Camera & Uncertainty & Right Camera & Uncertainty \\
\hline \multicolumn{5}{c}{ Focal length } \\
\hline$f_{x}$ & 1726.9 & 5.4966 & 1729.4 & 9.4974 \\
\hline$f_{y}$ & 1725.7 & 5.7818 & 1728.7 & 10.1684 \\
\hline \multicolumn{5}{c}{ Principal point } \\
\hline$u_{0}$ & 534.3812 & 3.7484 & 487.6526 & 6.8226 \\
\hline$v_{0}$ & 374.9889 & 1.4504 & 408.4091 & 2.6985 \\
\hline \multicolumn{5}{c}{ Distortion coefficients $k_{1,2}$ for radial and $p_{1,2}$ for tangential } \\
\hline$k_{1}$ & -0.3953 & 0.0047 & -0.4062 & 0.0163 \\
\hline$k_{2}$ & 0.1659 & 0.0605 & 0.2151 & 0.3397 \\
\hline$p_{1}$ & 0.0009 & 0.0000 & -0.0019 & 0.0001 \\
\hline$p_{2}$ & -0.0003 & 0.0008 & -0.0010 & 0.0015 \\
\hline
\end{tabular}

3.1.2 PST experimental setup The cameras used in this technique were two AVT Marlin F-145C 2 with IEEE 1394 IIDC FireWire interface with a resolution of 1280 x 960 pixels, and a laser beam to project a dot pattern, not necessary in this technique; however, for simplicity this work decided to use the laser to detect correspondence points between cameras as quick as possible.

Figure 2(a) shows the setup with 2 cameras at approximately an angle of $29^{\circ}$ between the optical axis of the cameras and the bar that holds the set up, and a laser that projects a point pattern over the measured gauge. Figure 2(b) shows the target point at the central part of the gauge block and the points on the origin plane. Figure 2(c) displays the correspondence points projected onto gauges and observed with both cameras. The matching of the points for each image of the left and right cameras generates an arrangement of points that represents the height of the gauge blocks and the height of the pyramid between the first level and the top level.

The resulting 3D reconstruction depends on image quality. In order to obtain images of sufficient quality we acquired experimental images under good illumination conditions as mentioned in (Belhaoua, 2010) and (Muquit, 2006).

We fitted a plane $\pi: a x+b y+c z+d=0$ and a point $p_{1}=$ $\left(x_{1}, y_{1}, z_{1}\right)$ outside the plane to calculate the measurements. The shortest distance from $p_{1}$ to plane is defined in Equation5:

$$
D=\frac{\left|a x_{1}+b y_{1}+c z_{1}+d\right|}{\sqrt{a^{2}+b^{2}+c^{2}}}
$$

where $a, b, c$ and $d$ are the coefficients of the plane and $\left(x_{1}, y_{1}, z_{1}\right)$ represents a point outside the plane.

3.1.3 Results of PST We tested PST using $3 \mathrm{~mm}, 50 \mathrm{~mm}$ and $100 \mathrm{~mm}$ gauge blocks and the fine-grained 3D printed piece 


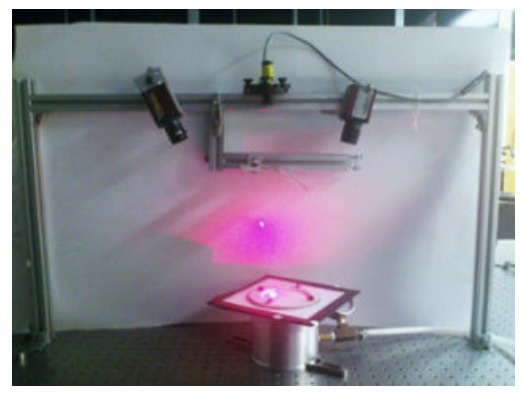

(a) Stereoscopy system

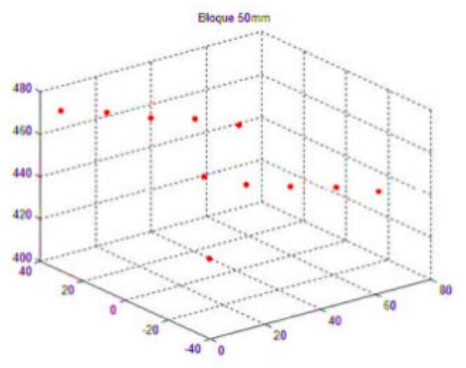

(b) 3D Points $(\mathrm{mm})$.

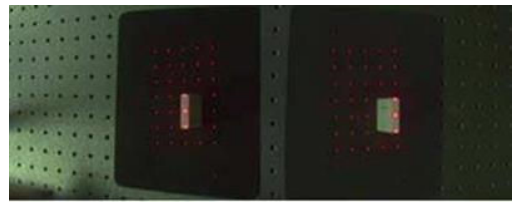

(c) Dots projection over a gauge block of $50 \mathrm{~mm}$.

Figure 2. Passive stereoscopy setup.

of gypsum (Pyramid). Based on (Hocken, 2011), the main factors influencing the accuracy of a stereovision system are as follows: defects of optical systems, errors caused by image processing, quality of the target, arrangement of the cameras, calibration errors, thermal error and stability camera mounting. The reported errors in Table 2 occurred due to different factors:

- Manual selection of correspondence points detected in both cameras, see Figure 2.

- The quality projection of the points is low.

- The angle arrangement of the cameras to the normal of the plane is less than $45^{\circ}$.

Table 2. Results of Passive Stereoscopy Technique.

\begin{tabular}{ccccc}
\hline Value $(\mathrm{mm})$ & Block 3 & Block 50 & Block 100 & Pyramid 15 \\
\hline True value & 3.000 & 50.000 & 100.000 & 15.081 \\
\hline Average & 2.848 & 47.776 & 91.846 & 13.455 \\
\hline Error & -0.152 & -2.224 & -8.154 & -1.626 \\
\hline$\sigma$ & 0.208 & 3.451 & 0.501 & 0.474 \\
\hline $\mathrm{U} k=2$ & 0.186 & 3.086 & 0.448 & 0.424 \\
\hline
\end{tabular}

The technique shows measurement errors greater than $2 \mathrm{~mm}$ when measured dimension exceeds $50 \mathrm{~mm}$ which is higher than the reported in the literature (Belhaoua, 2010). As a result, this technique seems to be unsatisfactory for measuring dimensions over $50 \mathrm{~mm}$. Table 2 shows that the increasing size of the gauge is directly proportional to the average error.

\subsection{Active Stereoscopic Technique (AST).}

One of the most popular active illumination devices is a laser or light stripe, which sweeps a plane of light across the scene or object while observing it from an offset viewpoint (Rioux, 1993) and (Curless, 1996), see Figure 3.

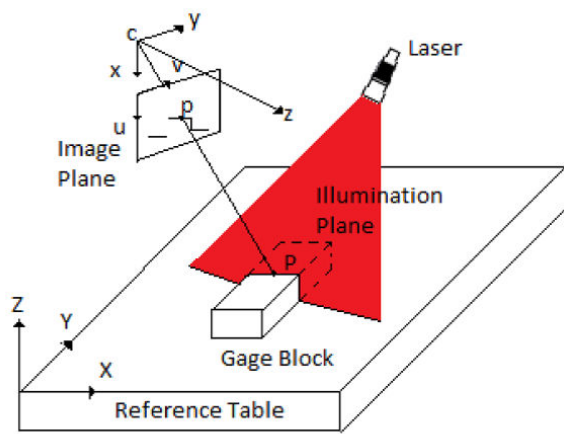

Figure 3. Illumination plane for 3D reconstruction with active stereoscopy.

The laser and the offset viewpoint (commonly a camera) enclose the Active Stereoscopic Technique (AST). As the stripe falls across the object, it deforms according to the shape of the surface it is illuminating. Then using optical triangulation we estimate the 3D locations of all the points seen in a particular stripe. In more detail, knowledge of the 3D plane equation of the light stripe allows us to infer the 3D location corresponding to each illuminated pixel. The accuracy of light striping techniques improves by finding the exact temporal peak in illumination for each pixel (Curless, 1996).

3.2.1 Calibration method and model for AST As in PST, the employed model is the pinhole, just the way to calculate extrinsic parameters is different. Table 1 shows right camera intrinsic parameters and its uncertainties. For this method is necessary to obtain the equation of the line-plane from the laser beam depicted in Figure 3. This work used four heights: $3 \mathrm{~mm}, 50 \mathrm{~mm}$, and $100 \mathrm{~mm}$ gauge blocks, and the reference plane. We fit four points from the top of these gauge blocks and 400 points from the reference plane outside the gauge blocks, giving a total of 404 points used to determine the plane of the line.

3.2.2 AST experimental setup Figure 4(a) shows the setup used on this technique. This setup was extracted from the PST setup depicted in Figure 2(a) using only the right camera and the laser.

In total, fifteen planes must be found equivalent to firteen lines according to the pattern of light generated by the laser (see Figure 4(a)). However we only need to find the plane equation of the line that the experimental setup from Figure 4(a) allows passing.

This plane will lead to the dimensional reconstruction of gauges blocks, and allows the determination of the depth of the object from the camera, see Figure 3.

3.2.3 Results of AST We used $3 \mathrm{~mm}, 50 \mathrm{~mm}$ and $100 \mathrm{~mm}$ length gauge blocks to test AST. Table 3 shows results calculated using fitted lines adjusted to the reference plane and a maximum point found on the peak of the signal see Figure 5.

According to Table 3, the average errors measured with this technique have a positive sign, this was because in the calibration 


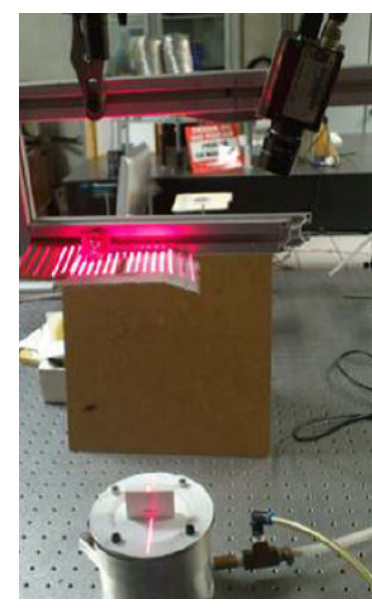

(a) Experimental setup for Active Stereoscopy.

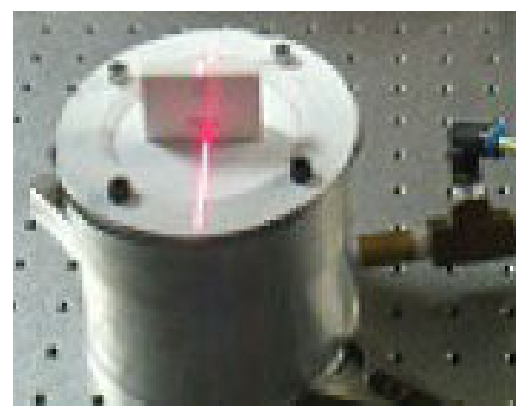

(b) Online 3D reconstruction of some measured gauges.

Figure 4. Active Stereoscopic Technique setup and measurement.

Table 3. Results of active stereoscopy technique.

\begin{tabular}{cccc}
\hline Value $(\mathrm{mm})$ & Block3 $\mathrm{mm}$ & Block $50 \mathrm{~mm}$ & Block $100 \mathrm{~mm}$ \\
\hline True value & 3.000 & 50.000 & 100.000 \\
\hline Average & 3.537 & 50.232 & 101.848 \\
\hline Error & 0.537 & 0.232 & 1.848 \\
\hline$\sigma$ & 0.021 & 0.007 & 0.004 \\
\hline $\mathrm{U} k=2$ & 0.019 & 0.006 & 0.003 \\
\hline
\end{tabular}

procedure we used the same printed pattern like in PST, but AST only use the right camera, which according to Table 2 does not have the same left camera intrinsic parameters (focal length, principal point and distortion). The highest standard deviation according to Table 3 is $0.021 \mathrm{~mm}$; this was due to the thickness of the light used. It generates noise that affects the image processing of pieces with dimensions less than $3 \mathrm{~mm}$, see Figure 5. Furthermore, the surface material where the light beam is projected is a factor that contributes to noise. Table 3 also shows the average error for the $50 \mathrm{~mm}$ and $100 \mathrm{~mm}$ gauges blocks increases from $0.232 \mathrm{~mm}$ to $1.848 \mathrm{~mm}$ proportional to its size, it is proposed to use a correction factor (usually linear) that change with the dimensions of the piece to be measured. This technique consists on a triangulation principle between the laser transmitter, the measured point and the camera. (Hocken, 2011) mentioned that the measured results of this technique depend heavily on the structure and angle of inclination of the surface (see Figure 3 ). This leads to relatively large measuring uncertainties making the use of this technique suitable only for less demanding applications. (Hocken, 2011) mentioned that better results can

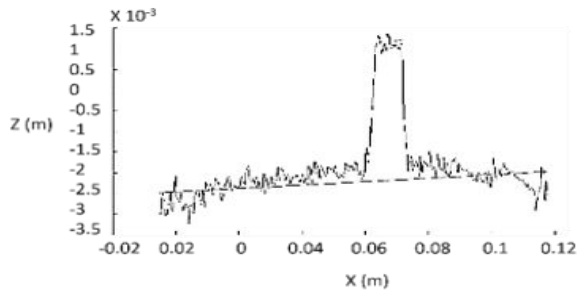

(a) $3 \mathrm{~mm}$ gauge block measure.

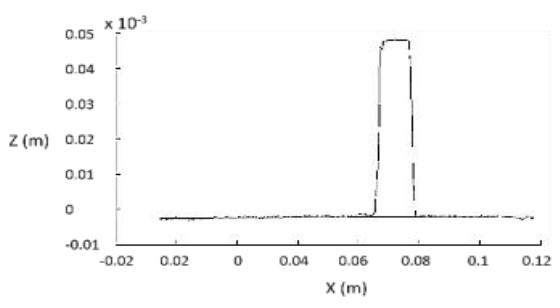

(b) $50 \mathrm{~mm}$ gauge block measure.

Figure 5. 2D reconstructions of gauge blocks using AST.

be achieved with laser sensors working according to the Focault principle.

\subsection{Shape from Contour Technique (SCT)}

(Baumgart, 1974) introduced the idea of using contours for 3D reconstruction. Afterwards (Szeliski, 1993) proposed a noninvasive digitizer built using a rotary table and a single camera with the reconstruction procedure of Shape from Silhouette (SFS) or Shape from Contour (SFC). SFS has become one of the most popular methods for the reconstruction of static objects (Gonzalez-Barbosa, 2015), (Fitzgibbon, 1998) and (Fang, 2003). SFC presents results with errors for objects of high complexity or detailed (Rodríguez, 2008).

3.3.1 Calibration method and model for SCT We based on (Gonzalez-Barbosa, 2015). The orthographic geometry illustrated in Figure 6, is the projection of a three dimensional point $Q(X, Y, Z)$ over a plane image generates a point $q(u, v)$ .Where $u=X$ and $v=Y$. Then, the model Equation 6 of orthographic projection in homogeneous coordinates $[u, v, 1]^{T}$ of a point $[X, Y, Z, 1]^{T}$ is

$$
q=K E Q
$$

where $K$ is defined as the matrix of intrinsic parameters, and $E$ as the matrix of extrinsic parameters, see Equation7

$$
K=\left(\begin{array}{cccc}
\alpha & \gamma & 0 & 0 \\
0 & \beta & 0 & 0 \\
0 & 0 & 0 & 0
\end{array}\right) ; E=\left(\begin{array}{cc}
R & T \\
0 & 1
\end{array}\right)
$$

The elements of $K$ include: $\alpha$ and $\beta$, the scale factors in image $u$ and $v$ axes, and $\gamma$ the parameter describing the skewness of the two image axes. The matrix $E$ represents the rigid transformation that carries the world coordinate system to the image coordinate system, composed of a rotation $\mathbf{R} \in R^{3 x 3}$ and a translation $\mathbf{T}$ $\in R^{3}$.

The calibration is carried out using the technique shown in (Fitzgibbon, 1998). The matrix of intrinsic parameters is denoted by: 


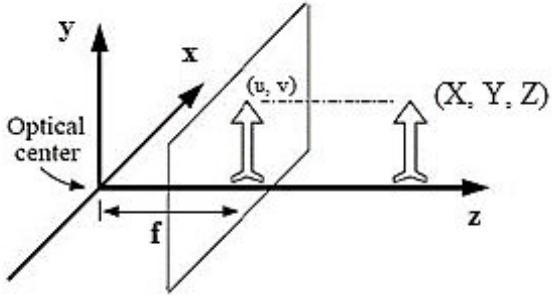

Figure 6. Orthographic projection model of a real object into the image plane.

$$
K=\left(\begin{array}{cccc}
14.325 & 0 & 0 & 0 \\
0 & 15.533 & 0 & 0 \\
0 & 0 & 0 & 1
\end{array}\right)
$$

The Equation used to convert the data from 2D image coordinates to $3 \mathrm{D}$ world coordinates is defined by:

$$
Q=E^{(-1)} K^{(-1)} q
$$

where $Q$ is a $3 x 1$ vector representing $3 \mathrm{D}$ world coordinates and $q$ is a $3 x 1$ vector of each pixel from the subpixelic contour. The Shape reconstruction From the contour technique was tested using $3 \mathrm{~mm}, 50 \mathrm{~mm}$ gauge blocks and the $10 \mathrm{~mm}$ cylinder gauge.

3.3.2 SCT experimental setup The SFC system used a telecentric lens (OPE TC 1364 by Opto Engineering), a turntable with a high accuracy encoder and 2.16 arc sec resolution (CR1Z7 by Thorlabs), and calibration pattern (PT 036-0596 by Opto Engineering), depicted in Figure 7.

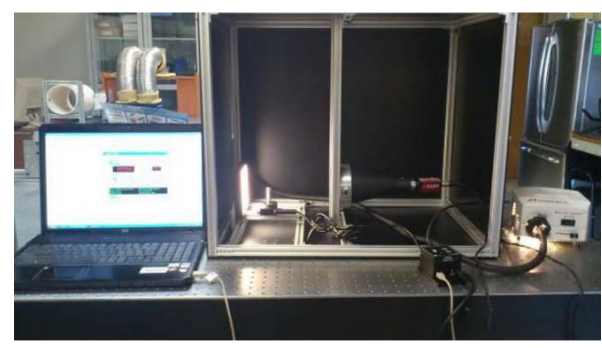

Figure 7. Shape from Contour setup.

Images of a rotating object illuminated from its back side are rendering. Consequently, the acquisition system can only see the contour of the object, as shown in Figure 8. We took 73 images with 5 degrees steps, $i=0,5, \ldots, 360$.

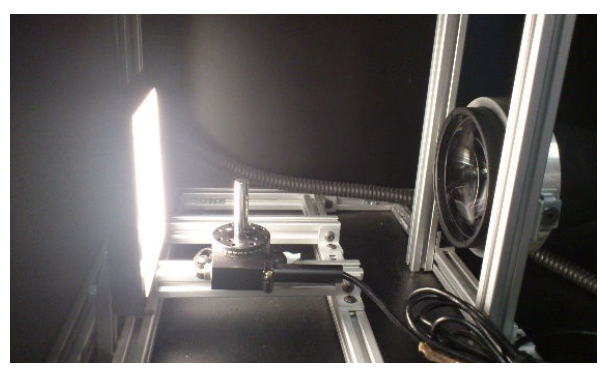

Figure 8. Cylinder images acquisition.

After the image acquisition, we applied a sub pixel extractor. The angle of rotation was calculated as the product of the image index with the rotation angle indicated by the turntable.
Using only one axis of rotation in all the images leads to inaccurate results. This inaccuracy is due to slight changes of the axis of rotation coming from illumination fluctuations and misalignment of the work piece with respect to the rotation of the turntable and the position of the camera. A dynamic rotation axis is necessary to reduce this inaccuracy. The first degree polynomial is a straight line fitted to all points over the subpixel contour image. This Equation is of the form:

$$
A_{u}+B_{v}+C=0
$$

where $u$ and $v$ are pixel coordinates and $A, B, C$ are the line coefficients.

Once calculated the dynamic axis of rotation, we have to perform a translation and rotation (eccentricity and leveling of the work piece with respect to the camera) of the coordinate system in pixel coordinates to the dynamic axis of rotation. This axis of rotation allows the 3D reconstruction with the accuracy showed in Table 4.

Table 4. Results of Shape from Contour Technique.

\begin{tabular}{cccc}
\hline Value $(\mathrm{mm})$ & Block 3 mm & Block 50 mm & Cylinder 10 mm \\
\hline True value & 3.000 & 50.000 & 10.000 \\
\hline Average & 2.924 & 49.887 & 9.785 \\
\hline Error & -0.076 & -0.113 & -0.215 \\
\hline$\sigma$ & 0.007 & 0.006 & 0.040 \\
\hline $\mathrm{U} k=2$ & 0.008 & 0.007 & 0.046 \\
\hline
\end{tabular}

At the same time, each one of the contour sub-pixel images was rotated around the $\mathrm{X}$ axis, and the gauge was plotted to show in real time the 3D reconstruction of the gauge, see Figure 9. Finally, the pixel coordinates were transformed to world coordinates using Equation 8.

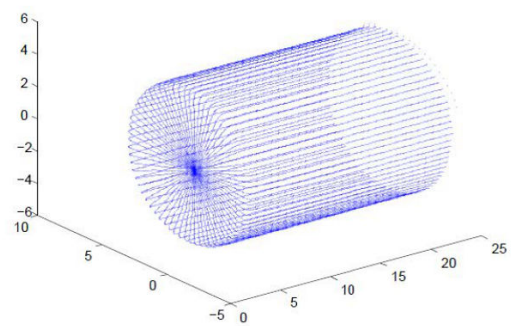

Figure 9. 3D reconstruction of $10 \mathrm{~mm}$ cylinder gauge.

3.3.3 Results of SCT In this technique we measured gauges three times. The measurement system acquired 73 images every $5^{\circ}$ degrees in $360^{\circ}$, equivalent to one and half hour of capture. In Table 4 we show an average error of $-0.215 \mathrm{~mm}$ in the cylinder of $10 \mathrm{~mm}$ diameter, this is due to the blurred contour of the gauge coming from reflections over cylinder metallic surface (see Figure 8 affecting the measurement uncertainty, conversely well-defined contours for the ceramic gauge blocks. According to (Hocken, 2011), this technique is suitable to measure flat objects. On the other hand, where wide edges are involved, the interrelationship among illumination, the test object, and the imaging beam path must be taken into consideration in the uncertainty calculation. Important elements that contributed to the low uncertainty and repeatability of this technique were telecentric lens, turntable, and calibration pattern. Despite errors in images processing caused by surface structures, pits, and contamination 
the technique provided better repeatability and lower uncertainty than AST and PST.

\subsection{Fringe Profilometry Technique (FPT)}

This technique for 3D reconstruction uses fringe projection. Exist several algorithms for processing the acquired images and unwrapping the phase, such as Fourier profilometry, phase shifting profilometry and temporal unwrapping profilometry technique, these are mentioned in (Rodriguez, 2008), (Takeda, 1983), (Villa, 2012) and (Cogravve, 1999).

We choose the temporal unwrapping profilometry technique tested in (Huntley, 1997) and (Xiao, 2010) because it is immune to noise and big gradients in test objects. It consists on projecting fringes with different periods at shifted phase; in our work we use, four images $I_{i}$ shifted 90 degrees at $t=1,2, \ldots, 7$ different frequencies.

The wrapped phase map for the value of $t=1$ is calculated with the Equation 10 for phase shifting.

$$
\phi_{w}(t)=\tan ^{-1}\left(\frac{I_{4}-I_{2}}{I_{1}-I_{3}}\right)
$$

For the next phase maps, we use the Equation 11

$$
\Delta \Phi_{w}(i, j)=\arctan \left(\frac{\Delta I_{42}(t) \Delta I_{13}(j)-\Delta I_{13}(t) \Delta I_{42}(j)}{\Delta I_{13}(t) \Delta I_{13}(j)+\Delta I_{42}(t) \Delta I_{42}(j)}\right)
$$

where

$$
\Delta I_{k l}(t)=I(k, t)-I(l, t)
$$

Finally, to find the unwrapped phase U, we apply the recursive equation with two consecutive wrapped phase maps. Equation 13

$$
U\left[\Phi_{1}, \Phi_{2}\right]=\Phi_{1}-2 \pi N I N T\left(\frac{\Phi_{1}-\Phi_{2}}{2 \pi}\right)
$$

With the value of the unwrapped phase, we can perform the calibration to calculate the metric values of the test object.

3.4.1 FPT calibration We performed the system calibration in two stages according to (Villa, 2012), the first one is the axial calibration which consisted in take 5 planes in $Z$ axis direction with known increments $\Delta Z$ (in this case $10 \mathrm{~mm}$ ). We project fringes according to (Cogravve, 1999) and (Huntley, 1997) on every plane and calculate a $4^{\text {th }}$ degree polynomial regression with the phase values in every plane at the known $Z$ positions.

Once we have this relationship, we proceed with axial calibration, to this we used a grid pattern with a $2 \mathrm{~cm}$ period and a bright dot in the point that we assume as the origin, see Figure 10. When the calibration is finished, we put the calibration gauges on the reference plane, and project fringes as showed at Figure 11.

3.4.2 FPT experimental setup. The experimental set up consisted of a Marlin F-080 CCD camera, a digital projector SONY VPL-EX5, both $1024 x 768$ pixels resolution, a flat surface and a millimetric rail shown in Figure 12.

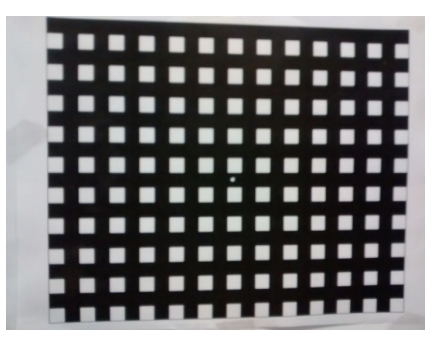

Figure 10. Grid pattern with $2 \mathrm{~cm}$ period.

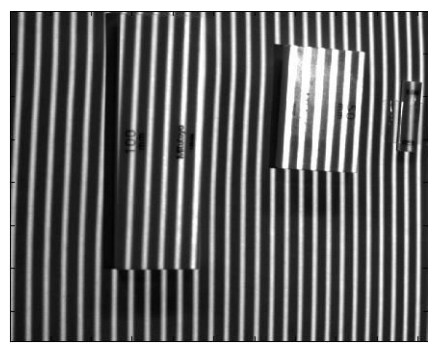

Figure 11. One of the acquired images.

3.4.3 Results of FPT Pyramid and calibration gauges reconstruction is showed at Figure 13 and 14.

Table 5 shows standard deviations less than $0.107 \mathrm{~mm}$ which demonstrate repeatability of the instrument. The average error increases with the time of use (about one hour) due to projector heating.

\section{COMPARATIVE RESULTS BETWEEN TECHNIQUES}

Figure 15 shows the measurement error of different gauges for the implemented technique. SFC has the best accuracy compared with the other three implemented techniques.

As shown in Figure 15, the average error in PST, AST and FP grows according to the size of the gauge because the viewing angle of the camera changes with the height of the measured piece; this suggests a correction of the measuring points with respect to the reference surface.

Figure 16 shows the uncertainties of the implemented techniques. In this Figure we can see that AST, SFC and FP are comparable, in these measurement systems the uncertainty decreases when the size of the piece is increasing. These systems show low repeatability to reconstruct pieces less than $3 \mathrm{~mm}$ in size. SFC also reduces uncertainty by increasing the size of the piece, without breaking its measurement range.

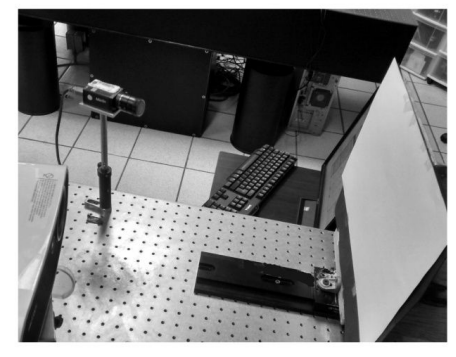

Figure 12. Experimental setup, we used a graduated rail to make the $10 \mathrm{~mm}$ increments. 


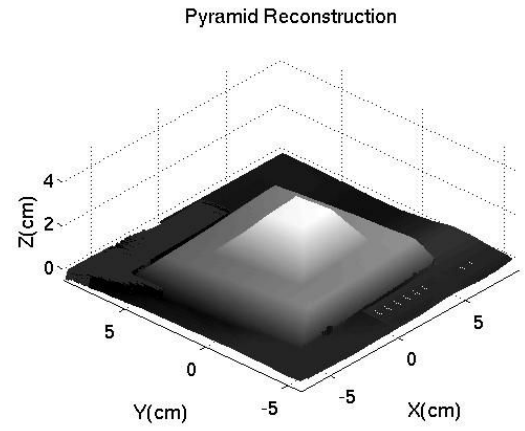

Figure 13. 3D reconstruction of pyramid, axis are at centimeters.

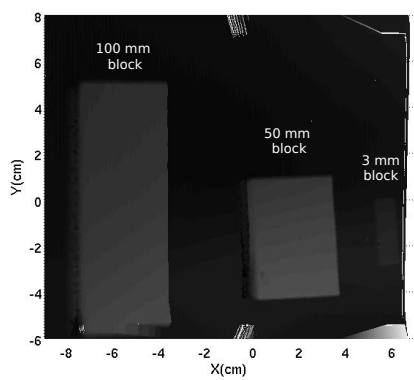

Figure 14. 3D reconstruction of the blocks, axis are at centimeters.

Uncertainties values of PST are out of scale with respect to the values of the other techniques in Figure 16.

\section{CONCLUSIONS AND DISCUSSIONS}

According with (Hocken, 2011) all of the implemented techniques can be improved in the calibration process if consider: image processing algorithms, temperature, intensity of illumination, optical magnification, imaging optics, telecentricity, camera resolution, edge detection technique, material of reference, reference surface tilt, optical magnification of laser sensor, scanning speed and laser intensity.

The software used to fit the points captured by any of the implemented techniques have a greatly influence on the quality of the measured results. We recommend use software with a calibration certificate. SFC, AST and PF, average errors, standard deviations, and uncertainties obtained with the present approach can help the user to identify the tolerances that each technique can achieve and to determine if these techniques can be applied to the control of some quality process or measuring process.

The performance of PST shows poor results for the tolerances that were expected based on (Belhaoua, 2010). Because of this, it is bad for quality control purposes, unless improvements in the factors considered on results of PST are done. Tables 6 and 7 describe the advantages and disadvantages of each technique. These

Table 5. Results of Fringe Profilometry Technique.

\begin{tabular}{ccccc}
\hline Value $(\mathrm{mm})$ & Block 3 & Block 50 & Block 100 & Pyramid 15 \\
\hline True value & 3.000 & 50.000 & 100.000 & 15.081 \\
\hline Average & 2.848 & 51.919 & 102.913 & 14.315 \\
\hline Error & -0.152 & 1.919 & 2.913 & -0.766 \\
\hline$\sigma$ & 0.098 & 0.088 & 0.028 & 0.107 \\
\hline $\mathrm{U} k=2$ & 0.070 & 0.044 & 0.016 & 0.050 \\
\hline
\end{tabular}

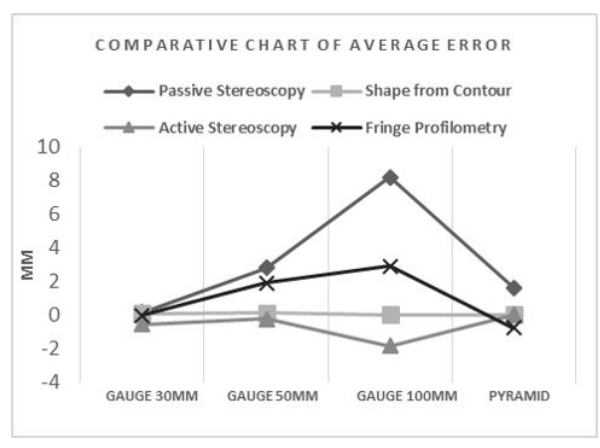

Figure 15. Comparative chart for average errors. $\mathrm{X}$-axis shows the measured objects and $\mathrm{Y}$-axis the average error in $\mathrm{mm}$.

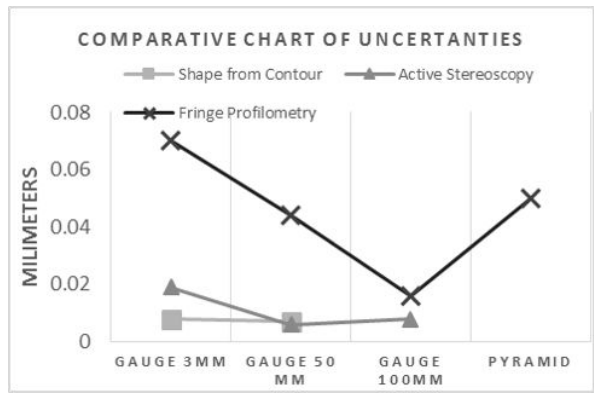

Figure 16. Comparative chart for uncertainties. $\mathrm{X}$-axis shows the measured objects and Y-axis the uncertainties at $\mathrm{mm}$.

tables let the user to take a decision. For example we can say that PST can be used on computer vision implementations, like robot navigation, but SFC, AST and FP can be used on metrological issues, like reverse engineering.

To calibrate the cameras in PST, AST and FP techniques, we used a printed pattern, this implies an error in the calibration results which affects the error of measurement. The printed patterns introduce an error that can be considered systematic according to (Xiao, 210) and therefore corrected, however, the residuals once the correction is applied, must be part of the uncertainty measurement.

Also the calibration process must take into consideration factors such as temperature of the structure holding the cameras, flatness where the printed pattern are pasted, humidity, image processing and others. These and other errors can have a systematic nature and can be corrected, improving the measurement error. These corrections influence the error measurement uncertainty .

The method used by the SFC technique is very different from PST, AST and FP techniques where a pinhole model is considered. The model used was based on (Gonzalez-Barbosa, 2015); however, we must consider that the model could have other errors not considered like the heating of the screen light from Figure 7. In its favor, this technique uses a certificate pattern instead of a printed one.

Future work will consider the influence of the above errors for smaller measurement error and find major factors of uncertainty affecting the measurements.

\section{ACKNOWLEDGMENT}

The authors thank Consejo Nacional de Ciencia y Tecnologa (CONACYT) for the scholarships awarded (227445, 339890 and 
Table 6. Reconstruction techniques PST and AST with advantages and disadvantages of our implemented systems.
Table 7. Reconstruction techniques SCT and FPT with advantages and disadvantages of our implemented systems.

\begin{tabular}{|c|c|}
\hline \multicolumn{2}{|c|}{ Passive Stereoscopy Technique (PST) } \\
\hline Advantages & Disadvantages \\
\hline $\begin{array}{l}\text { - Can be set to objects } \\
\text { of different sizes at } \\
\text { different distances in } \\
\text { various environmental } \\
\text { conditions. } \\
\text { - Did not has correspon- } \\
\text { dence problems when } \\
\text { a dot pattern was pro- } \\
\text { jected onto the sur- } \\
\text { face, these projected } \\
\text { points simplified the } \\
\text { calculation of its 3D } \\
\text { position. } \\
\text { - Approximate cost: } \\
\$ 1,101.98 \text { USD. }\end{array}$ & $\begin{array}{l}\text { - In the absence of a pro- } \\
\text { jection pattern; a point in } \\
\text { the image of one of the } \\
\text { cameras can be associated } \\
\text { with several points on the } \\
\text { image of the other camera } \\
\text { (false match) } \\
\text { - If exits a hide point by } \\
\text { occlusions in the field } \\
\text { of view of one cameras } \\
\text { maybe in the other field of } \\
\text { view of the second camera } \\
\text { this point are visible. } \\
\text { - The selection of corre- } \\
\text { spondence points was } \\
\text { made manually can cause } \\
\text { mismatches errors. }\end{array}$ \\
\hline \multicolumn{2}{|c|}{ Active Stereoscopy (AST) } \\
\hline Advantages & Disadvantages \\
\hline $\begin{array}{l}\text { - Currently used in sev- } \\
\text { eral commercial mea- } \\
\text { surement systems. } \\
\text { - Presented robustness } \\
\text { to measurements } \\
\text { made surfaces without } \\
\text { texture. } \\
\text { - To establish the re- } \\
\text { lationship between } \\
\text { the 2D image points } \\
\text { and points in the } \\
\text { 3D world, the tri- } \\
\text { angulation method } \\
\text { used. } \\
\text { - Approximate cost: } \\
\$ 1,689.99 \text { USD. }\end{array}$ & $\begin{array}{l}\text { - One of the main limita- } \\
\text { tions of this technique is } \\
\text { the problem of occlusion, } \\
\text { which occurs when the } \\
\text { camera is not able to dis- } \\
\text { play the laser beam re- } \\
\text { flected by the object, since } \\
\text { the object geometry. } \\
\text { - The camera reaches de- } \\
\text { tect unwanted reflections } \\
\text { generated by the geomet- } \\
\text { ric and physical character- } \\
\text { istics of the object. } \\
\text { - The reflected laser beam } \\
\text { on the object has a thick- } \\
\text { ness and this introduces } \\
\text { an impression when de- } \\
\text { tecting the light beam in } \\
\text { the image acquired by the } \\
\text { camera. } \\
\text { - The laser power affects the } \\
\text { sharpness of this on pur- } \\
\text { pose, because if the in- } \\
\text { tensity is not enough, the } \\
\text { plane of light will not be } \\
\text { detected in the image. }\end{array}$ \\
\hline
\end{tabular}

\begin{tabular}{|c|c|}
\hline \multicolumn{2}{|c|}{ Shape from contour (SCT) } \\
\hline Advantages & Disadvantages \\
\hline $\begin{array}{l}\text { - Allows rebuild small } \\
\text { objects, up to } 5 \mathrm{~cm} \text {. } \\
\text { - When using telecen- } \\
\text { tric lenses, the error is } \\
\text { eliminated in perspec- } \\
\text { tive vision systems, so } \\
\text { that objects can zoom } \\
\text { in and out of the cham- } \\
\text { ber without changing } \\
\text { the image dimensions. } \\
\text { - System Approximate } \\
\text { cost: } \$ 3,454.99 \text { USD }\end{array}$ & $\begin{array}{l}\text { - The cost and size of the } \\
\text { telecentric lens are in- } \\
\text { versely proportional to the } \\
\text { size of the object being } \\
\text { measured. } \\
\text { - When using a lighting sys- } \\
\text { tem light behind the object } \\
\text { to be measured, the cam- } \\
\text { era can detect reflections } \\
\text { produced by the geomet- } \\
\text { rical and physical charac- } \\
\text { teristics of the material, } \\
\text { making the object appear } \\
\text { smaller from the actual } \\
\text { size. } \\
\text { - Lighting should be con- } \\
\text { trolled, as excess or defi- } \\
\text { ciency camera is blinded. } \\
\text { - The telecentric lenses are } \\
\text { much more expensive than } \\
\text { conventional lenses, used } \\
\text { in the other techniques. } \\
\text { - If the object surface has } \\
\text { concave vertical curves, } \\
\text { you will not be recon- } \\
\text { structed and the recon- } \\
\text { struction system fill in } \\
\text { these areas. }\end{array}$ \\
\hline
\end{tabular}

\begin{tabular}{|c|c|}
\hline \multicolumn{2}{|c|}{ Fringe Profilometry Technique (FPT) } \\
\hline Advantages & Disadvantages \\
\hline $\begin{array}{l}\text { - Fast and easy imple- } \\
\text { mentation. } \\
\text { - Allows accurate and } \\
\text { dense measurements } \\
\text { on large objects. } \\
\text { - Robust to noises and } \\
\text { sharp edges. } \\
\text { - Gives entire height } \\
\text { distribution at every } \\
\text { pixel. } \\
\text { - Approximate cost: } \\
\$ 1000.00 \text { USD. }\end{array}$ & $\begin{array}{l}\text { - Lighting should be con- } \\
\text { trolled, in excess or defi- } \\
\text { ciency camera is blinded. } \\
\text { - If exist shadows or occlu- } \\
\text { sions the 3D shape is loss. } \\
\text { - Dependent on projector } \\
\text { power and resolution. } \\
\text { - For the implementation } \\
\text { presented here, requires } \\
\text { many images. }\end{array}$ \\
\hline
\end{tabular}

371999), Instituto Politécnico Nacional (IPN) particularly at Centro de Investigación en Ciencia Aplicada y Tecnología Avanzada Unidad Querétaro (CICATA-Qro) for the financial support given, and Centro Nacional de Metrología (CENAM) for the facilities granted. 


\section{REFERENCES}

Baumgart B., 1974. Geometric Modeling for Computer Vision. PhD Thesis, Stanford University.

Belhaoua A., Kohler S. and Hirsch E., 2010. Error Evaluation in a Stereovision-Based 3D Reconstruction System. EURASIP Journal on Image and Video Processing pp. 1-12.

Bouguet, J. Y., 2013. Camera calibration toolbox for MatLab. Last updated December 2n̂d, 2013.

Bolles R. C., Kremers J. H. and Cain R. A., 1981. A simple sensor to gather three-dimensional data. Technical note 249, SRI.

Coggrave C.R. and Huntley J.M., 1999. High-speed surface profilometer based on a spatial light modulator and pipeline image processor. Opt. Eng., 38(9), pp. 1573-81.

Curless B., and Levoy M., 1996. A volumetric method for building complex models from range images. In ACM SIGGRAPH 1996 Conference Proceedings, pp. 303312, New Orleans.

Fang Y. H., Chou H. L. and Chen Z., 2003. 3D shape recovery of complex objects from multiple silhouette images. Pattern Recognition Letters, 24(9-10) pp. 12791293.

Fitzgibbon A. W., Cross G. and Zisserman A., 1998. Automatic 3D Model Construction for Turn Table Sequences. In Proceedings of the European Workshop on 3D Structure from Multiple Images of Large-Scale Environments, pp. 155-170.

Gonzalez-Barbosa J.J., Rico-Espino J.G., Gómez-Loenzo R.A., Jiménez-Hernández H., Razo M., Gonzalez-Barbosa M, 2015. Accurate 3D Reconstruction using a Turntable-based and Telecentric Vision. AUTOMATIKA, Vol. 56 (4), pp. 508521.

Evaluation of measurement data - Guide to the expression of uncertainty in measurement, JCGM 100:2008 GUM 1995 with minor corrections. First edition 2008 Corrected version, 2010.

Hall E. L., Tio B. K., JMcPherson C. A. and Sadjadi F. A., 1982. Measuring curved surfaces for robot vision. Comput. Vol. (15), pp. $42-54$.

Heikkila J. and Silve O., 1997. A four-step camera calibration procedure with implicit image correction. Proceedings IEEE CVPR, pp. 11061112.

Hocken R. J. and Pereira P. H., 2011. Coordinate Measuring Machines and Systems. 2nd Edition, CRC Press, USA, pp. 30, 129, 131, 134, 135, 149, 493, 494.

Huntley J.M. and Saldner H.O., 1997. Error-reduction methods for shape measurement by temporal phase unwarpping. Opt. Soc. Am.,14(12), pp. 3188-96.

ISO 14253-1, 1998. Geometrical Product Specifications (GPS) Inspection by measurement of work pieces and measuring equipment Part 1: Decision rules for proving conformance or nonconformance with specifications, Geneva.

ISO 5725-1/Cor 1, 1998. Geometrical product specifications (GPS) Accuracy (trueness and precision) of measurement methods and results Part 1: General principles and definitions.

ISO 15530-3, 2011. Geometrical product specifications (GPS) Coordinate measuring machines (CMM): Technique for determining the uncertainty of measurement Part 3: Use of calibrated work pieces or measurement standards.
Maier-Hein L., Mountney P., Bartoli A., Elhawary H., Elson D., Groch A., Kolb A., Rodrigues M., Sorger J., Speidel S. and Stoyanov D., 2013. Optical techniques for 3D surface reconstruction in computer-assisted laparoscopic surgery, Medical Image Analysis, vol. 17, pp. 974996.

Muquit M. A., Shibahara T. and Aoki T., 2006. A High-Accuracy Passive 3D Measurement System Using Phase-Based Image Matching. IEICE TRANS. FUNDAMENTALS, E89A(3).

Ng S. C. and Ismail N., 2011. Reconstruction of the 3D object model: a review, SEGi Review, Vol. 4 (2) pp. 18-30.

Rashmita K., Chitrakala Dr. S. and SelvamParvathy S., 2013. 3D Image Reconstruction: Techniques, Applications and Challenges. Proceedings of International Conference on Optical Imaging Sensor and Security, Coimbatore, Tamil Nadu, India.

Ramos B., Santos E., 2011. Comparative Study of Different Digitization Techniques and Their Accuracy. Computer Aided Design, Vol. 43 (2), pp. 188-206.

Rioux M., and Bird T., 1993. White laser, synced scan. IEEE Computer Graphics and Applications, 13(3), pp. 1517.

Rodríguez J., 2008. Técnicas de Reconstrucción Volumétrica a partir de Múltiples Cámaras: Aplicación en Espacios Inteligentes. Bachelor's Thesis, Universidad de Alcalá, Escuela Politécnica Superior, Ingeniería Electrónica.

Salvi J., Matabosch C., Fofi D. and Forest J., 2007. A review of recent range image registration methods with accuracy evaluation. Image and Vision Computing, Vol. 25 (5), pp. 578-596.

Szeliski R., 2010. Computer Vision: Algorithms and Applications. Springer, Chapter 11: Stereo correspondence, pp. 537-567, ISBN: 978-1-84882-935-0.

Szeliski R., 1993. Rapid octree construction from image sequences. Computer Vision Graphics and Image Processing: Image Understanding, Vol. 58(1) pp. 23-32.

Takeda M. and Mutoh K., 1983. Fourier transform profilometry for the automatic measurement of 3-D object shapes. App. Opt., 22(24), pp. 3977-3982.

Tsai R., 1987. A Versatile Camera Calibration Technique for High-Accuracy 3D Machine Vision Metrology Using Off-theshelf TV Cameras and Lenses. IEEE J. Rob. Autom. Vol. 3(4), pp. 323-44.

Vilaca J. L., Fonseca J. C. and Pinho A. M., 2010. Non-contact 3D acquisition system based on stereo vision and laser triangulation. Machine Vision and Applications, Vol. 21, pp. 341350.

Villa J., Araiza M., Alaniz D., Ivanov R. and Ortiz M., 2012. Transformation of phase to $(\mathrm{x}, \mathrm{y}, \mathrm{z})$-coordinates for the calibration of a fringe projection profilometer. Opt. Laser Eng., 50(2), pp. 256-261.

Xiao Y. and Fisher R. B., 2010. Accurate feature extraction and control point correction for camera calibration with a mono-plane target. Proc. 2010 Int. Conf. on 3D Processing, Visualization and Transmission. 